\title{
The impact of grain quantity on the biology of Sitophilus zeamais Motschulsky (Coleoptera: Curculionidae): oviposition, distribution of eggs, adult emergence, body weight and sex ratio
}

\author{
M. Danho*, C. Gaspar, E. Haubruge \\ Unit of Pure and Applied Zoology, Gembloux Agricultural University, 2, Passage des Déportés, \\ B-5030 Gembloux, Belgium
}

Accepted 8 June 2001

\begin{abstract}
Studies were conducted to observe the effect of grain quantity on the oviposition, distribution of eggs, adult emergence, adult body weight and sex ratio of Sitophilus zeamais, an important pest of maize. Three quantities of maize grains (200, 400 and 800 grains/glass jar of 11) were used under laboratory conditions $\left(30^{\circ} \mathrm{C}\right.$ and $70 \%$ r.h.). Twenty five male/female pairs of maize weevil were introduced into each glass jar for $12 \mathrm{~d}$. The greatest and lowest number of eggs was laid on batches of 800 and 200 grain kernels, respectively. The total number of grains attacked followed a similar trend. The aggregation of eggs expressed as a ratio of the variance to mean increased as the amount of maize grains was increased. The aggregation parameter $k$ ranged from 2.35 on 200 grains to 4.49 on 800 grains. On average, a significantly higher proportion of grains were infested $(90 \%)$ when less grain was present. Emergence of adult weevils was, however, maximal (472 individuals/glass jar) when the grain quantity was highest. The mean weight of the emerged adults was not significantly influenced by grain quantity, but female weevils were heavier than males (mean weight 3.16 vs. $3.05 \mathrm{mg}$ ) irrespective of maize quantity and eggs laid. Sex ratio (males/100 females) of emerged adults did not differ among treatments. The importance of grain quantity, and of oviposition, in internally feeding granivores and the possibility that there is an adaptive reproductive strategy are discussed. (C) 2002 Elsevier Science Ltd. All rights reserved.
\end{abstract}

Keywords: Sitophilus zeamais; Oviposition behaviour; Egg distribution; Grain quantity; Environmental influence

\footnotetext{
*Corresponding author. Tel.: + 32-81-622348; fax: + 32-81-622312.

E-mail address: zoologie@fsagx.ac.be,danho.m@fsagx.ac.be (M. Danho).
} 


\section{Introduction}

The maize weevil, Sitophilus zeamais Motsch. is one of the most serious cosmopolitan pest of stored cereal grain, especially of maize (Zea mays, L.), in tropical and sub-tropical regions (Throne, 1994). Farmers in Africa generally store their maize in open granaries. In recent years, post-harvest losses to storage insect pests such as the maize weevil, have been recognised as an increasingly important problem in Africa (Markham et al., 1994). Cheap and effective methods for reducing $S$. zeamais damage are needed in these countries.

A fundamental knowledge of the biology of $S$. zeamais is a prerequisite for devising methods of efficient control. In order to understand the biology of the maize weevil, a sound knowledge about its response to the effects of environmental and biological factors is essential. There have been many studies of the biology and behaviour of Sitophilus species, mostly on S. granarius and $S$. oryzae and these have been periodically reviewed by many authors (Richards, 1947; Segrove, 1951; Longstaff, 1981). Insect oviposition behaviour is an important contributor to the fitness of insects because of the consequent effect on the number and quality of offspring (Smith, 1986; Honek, 1993; Stejskal and Kucerova, 1996). Oviposition behaviour varies according to insect species and strain, population density, environmental conditions, food, age and size of the individual (Stejskal and Kucerova, 1996). There have been numerous reports on studies of interactions of $S$. zeamais with the environment. Some studies on population dynamics have been conducted minimising the number of maize grains (Schoonhoven et al., 1976; Widstrom et al., 1978) or number of weevils, or both (Dobie, 1974; Widstrom et al., 1978). Despite the importance of $S$. zeamais, there are not any recent quantitative data describing its life history traits over the range of environmental conditions at which it will develop. An understanding of the biology and behaviour of the maize weevil in relation to grain quantity will assist in the development of improved management practices for the control of this pest.

Therefore, the purpose of this work was to study oviposition, distribution of eggs, adult emergence, weight and sex-ratio of S. zeamais kept in three quantities of maize grain.

\section{Materials and methods}

\subsection{Insects and substrate}

Insect material used was originally collected in Togo (West Africa) and subsequently cultured in the laboratory on maize of the variety "Plata". Intact whole grains of uniform size were chosen for the experiment and equilibrated in a constant temperature and humidity (CTH) room at $30^{\circ} \mathrm{C}$ and $70 \%$ r.h. All of the experiments were carried out in the same CTH room in darkness. Weevils used were obtained from laboratory stocks, and the culturing techniques used have been described by Walgenbach et al. (1983). Dimorphic rostral characteristics (Halstead, 1963) were used to separate the sexes.

\subsection{Oviposition}

Three amounts of maize grain (200, 400 and 800 grain kernels) were put in 11 glass jars. For each quantity, two jars were set up every week for 4 weeks, making a total of 8 replicates per grain 
quantity. Twenty five male/female pairs of maize weevil, $<2 \mathrm{~d}$ old after emergence, were introduced into each glass jar which was placed and kept in the CTH room. Weevils were allowed to feed, mate and oviposit for $12 \mathrm{~d}$. After removing the adults, $10 \%$ of each treatment (that is 20 , 40 and 80 grains/jar) was dipped in acid-fuchsin to stain the egg plugs in order to count the eggs laid (Frankenfeld, 1948). It was assumed that each plug covered only one egg and each egg was covered by one plug. The method used to stain the grain was adapted after the report of Sharifi (1972) (the grains were immersed for approximately $60 \mathrm{~s}$ in water, then for $45 \mathrm{~s}$ into the stain and for at least $30 \mathrm{~s}$ in water for de-staining). After staining, excess water was removed from the grain's surface by drying with tissue paper. Jars were sampled on the same day when the parental adults were removed. Each stained grain was then examined separately with a binocular microscope and records were made as follows:

1. number of eggs laid,

2. number of grains infested,

3. frequency distribution of egg number per grain.

After these observations, sampled grains were put back in their original jars.

\subsection{Progeny production}

The jars containing infested maize were kept for 7 weeks in the CTH room after which the emerged adults were sieved off. This procedure simulates culture conditions in which the culture interval is 8 weeks, taking into consideration that mating in $S$. zeamais does not occur before weevils are $3 \mathrm{~d}$ old (Walgenbach and Burkholder, 1987). There was little possibility of third generation adults emerging since the minimum time needed for development is $28 \mathrm{~d}$ (Povey and Sibly, 1992). The weevils emerging from each jar were counted to give a measure of productivity. Then 100 individuals were randomly selected from each jar and sexed to determine the sex ratio. The sex ratio is defined as the proportion of males to females and is expressed as the number of males per 100 females (Richards, 1947). Differences in sex ratios among grain quantities were tested by comparing the proportions within and between quantities. The sexed weevils were weighed (using a Sartorius micro-balance) to evaluate differences according to sex.

\subsection{Statistical analysis}

\subsubsection{Oviposition}

The aggregation of eggs was expressed as the ratio of the variance to the mean $(V / M) . V / M$ ratio was used because it is a good indicator of clumping and its value is meaningful (Myers, 1978). Moreover, the $V / M$ ratio is only weakly correlated with the mean population density and has the advantage of being easy to compute and readily understandable (Myers, 1978). A $V / M$ ratio of 1.0 indicates a Poisson distribution: values that are significantly greater than 1.0 indicate aggregation (Legg et al., 1987). Such distributions have been adequately described by the negative binomial distribution, which is characterised by two parameters, the mean and the degree of clumping $k$. If a distribution is very clumped the $k$ value is small (less than 1). As the distribution becomes less clumped $k$ becomes larger; for a Poisson distribution $k$ approaches infinity. Thus, 
estimation of $k$ values indicates whether a distribution is random or shows degrees of clumping. One of the simplest methods for calculating a common $k$ is regression analysis (Holloway, 1984).

\subsubsection{Progeny production}

Mean number of emerged adults, mean body weight and sex ratios were compared by analysis of variance (ANOVA) based on the Minitab computer package. When the analysis of variance indicated that significant $(P<0.05)$ differences were found, means were separated by Tukey's test at $P \geqslant 5 \%$ level.

\section{Results}

\subsection{Oviposition}

\subsubsection{Number of eggs laid}

The numbers of eggs recorded in each grain quantity are given in Table 1 together with the number of grain infested by $S$. zeamais. With the different quantities sampled (20, 40, 80 grains/ jar), we scored a total of 609,713 and 863 eggs, respectively, for the eight replicates. The ANOVA indicated that there was a significant difference $(P<0.05 ; F=5.03 ; D F=2)$ between these three values. The number of eggs laid increased when the amount of available grain increased.

\subsubsection{Frequency of eggs per grain}

Table 2 shows the frequency distribution of egg number per grain in the three treatments. The $V / M$ ratio is $4.07,9.76$ and 82.74 for the low, the middle and the high quantity, respectively. The $V / M$ ratio increased with an increasing amount of maize grain. Considering that these three values were significantly greater than 1.0 , we concluded that aggregation of $S$. zeamais eggs occurred at each grain quantity. This conclusion is also supported by the $k$ values calculated. The weevils deposited eggs in an aggregated manner, since most of the egg plug distributions produced relatively small $k$ values (Table 2). The small $k$ values indicate that $S$. zeamais clumped egg plugs on the three quantities used. Table 2 shows the values in the frequency distribution of eggs per grain and also the expected values of the Poisson distribution.

Table 1

Effect of different grain quantities on egg number of Sitophilus zeamais and on the rate of kernel infestation ( $\%$ in brackets) of eight replicates/treatment ${ }^{\mathrm{a}}$

\begin{tabular}{lllr}
\hline $\begin{array}{l}\text { Number of sampled } \\
\text { grains/replicate }\end{array}$ & $\begin{array}{l}\text { Mean number of eggs laid } \\
\text { per replicate }\end{array}$ & \multicolumn{2}{l}{ Grain number per treatment } \\
\cline { 3 - 4 } & & Infested & Uninfested \\
\hline 20 & $76.13 \mathrm{a}$ & $145(90.63)$ & $15(9.37)$ \\
40 & $89.13 \mathrm{ab}$ & $246(76.87)$ & $74(23.13)$ \\
80 & $107.88 \mathrm{bc}$ & $400(62.50)$ & $240(37.50)$ \\
\hline
\end{tabular}

\footnotetext{
${ }^{a}$ Values with the same letter within a column are not significantly different, Tukey's HSD test, $P<0.05$.
} 
Table 2

The observed values of frequency distribution of Sitophilus zeamais eggs per grain compared with the expected values of a random distribution (Poisson series). Results are means of eight replicates $/$ treatment $^{\mathrm{a}}$

\begin{tabular}{|c|c|c|c|c|c|c|}
\hline \multirow[t]{3}{*}{ Eggs/grain } & \multicolumn{6}{|c|}{ Number of sampled grains/replicate } \\
\hline & \multicolumn{2}{|c|}{20} & \multicolumn{2}{|c|}{40} & \multicolumn{2}{|c|}{80} \\
\hline & (a) & (b) & (a) & (b) & (a) & (b) \\
\hline 0 & 15 & 6.44 & 74 & 36.47 & 240 & 169.05 \\
\hline 1 & 25 & 20.69 & 67 & 79.20 & 166 & 225.05 \\
\hline 2 & 26 & 33.24 & 57 & 86.01 & 120 & 149.80 \\
\hline 3 & 30 & 35.59 & 47 & 62.27 & 56 & 66.47 \\
\hline 4 & 11 & 28.58 & 27 & 33.81 & 27 & 22.12 \\
\hline 5 & 15 & 18.36 & 23 & 14.69 & 16 & 5.89 \\
\hline $6+$ & 38 & 9.83 & 25 & 5.32 & 15 & 1.31 \\
\hline Chi-square & \multicolumn{2}{|c|}{106.845} & \multicolumn{2}{|c|}{428.99} & \multicolumn{2}{|c|}{214.74} \\
\hline & \multicolumn{2}{|c|}{12.59} & \multicolumn{2}{|c|}{12.59} & \multicolumn{2}{|c|}{11.07} \\
\hline$V / M$ ratio & \multicolumn{2}{|c|}{4.07} & \multicolumn{2}{|c|}{9.76} & \multicolumn{2}{|c|}{82.74} \\
\hline$K$ value & \multicolumn{2}{|c|}{2.35} & \multicolumn{2}{|c|}{5.06} & \multicolumn{2}{|c|}{4.49} \\
\hline
\end{tabular}

${ }^{a}$ (a) number observed; (b) number expected; (c) value of Chi-square required for significance at $P=0.05$.

It can be seen that when many eggs were laid on the larger quantities of grains, the frequency of highly infested grains ( 3 or more eggs/grain) decreased from $14.69 \%$ with 3 eggs to $7.80 \%$ for 6 or more eggs with 400 grains and from 8.75 to $2.34 \%$, respectively, with 800 grains, although a great number of grains (23.13 and $37.5 \%$ for the middle and the high quantity, respectively) were still left uninfested.

\subsubsection{Percentage of grain infested}

In the low quantity treatment, females used a few more grains $(90 \%)$ compared to $77 \%$ in the middle quantity and $62.5 \%$ in the high quantity (Table 1). The number of uninfested grains was higher in the high quantity treatment and lower in the low quantity treatment. In any case, we noted that after $12 \mathrm{~d}$ of infestation, females had used more than $50 \%$ of the available grain regardless of the grain quantity available. The number of eggs laid per grain followed a similar trend.

\subsection{Progeny production}

\subsubsection{Adult emergence}

The effect of grain quantity on the population increase of $S$. zeamais is shown in Table 3 . The number of progeny increased in parallel with the quantity of maize provided. Significant differences $(P=0.0001<0.05, F=54.14, D F=2)$ were found between the numbers of emerged adults. The most adults emerged from the high quantity and the least from low quantity treatment. 
Table 3

Mean number $( \pm$ SE) of emerged adults, adult body weight $(\mathrm{mg})$ and sex ratio (males/100 females) of Sitophilus zeamais reared on eight replicates of three maize grain quantities ${ }^{\mathrm{a}}$

\begin{tabular}{|c|c|c|c|}
\hline & \multicolumn{3}{|c|}{ Number of grains/jar } \\
\hline & 200 & 400 & 800 \\
\hline Number of emerged adults & $153.38 \mathrm{c}( \pm 11.40)$ & $271.25 b( \pm 10.54)$ & $472.38 \mathrm{a}( \pm 37.73)$ \\
\hline Female weight & $3.15 \mathrm{~d} \quad( \pm 0.03)$ & $3.16 \mathrm{~d} \quad( \pm 0.03)$ & $3.17 \mathrm{~d} \quad( \pm 0.04)$ \\
\hline Male weight & $3.04 \mathrm{e} \quad( \pm 0.04)$ & $3.05 \mathrm{e} \quad( \pm 0.03)$ & $3.06 \mathrm{e} \quad( \pm 0.04)$ \\
\hline Sex ratio & $93.39 \mathrm{f} \quad( \pm 5.08)$ & $79.82 \mathrm{f} \quad( \pm 7.70)$ & $86.07 f( \pm 10.24)$ \\
\hline
\end{tabular}

${ }^{a}$ Values with the same letter within a row are not significantly different, Tukey's HSD test, $P<0.05$.

\subsubsection{Adult body weight}

There were no significant differences among the treatments regarding the adult weight (Table 3). Similarly, no significant differences were detected between the progeny's sex and the initial amount of maize kernels. However, a significant difference $(P=0.001<0.05, F=11.93, D F=1)$ was found between the weight of males and females. As expected, female weevils were heavier than males (mean weight 3.16 vs. $3.05 \mathrm{mg}$ ). The number of kernels did not influence the weight of males and females.

\subsubsection{Adult sex ratio}

The sex ratios for the treatment variables did not differ significantly from each other $(P=0.391>0.05 ; D F=2 ; F=0.98)$. The mean sex ratio expressed as males per 100 females in the treatments varied from 79.8 with 400 grains to 93.4 with 200 grains (Table 3). There were always less males than females, but no difference was significant as judged by the Tukey's HSD test.

\section{Discussion}

This study dealt with stored product pests whose immature stages live and feed within a grain. This kind of pest is faced with special evolutionary problems. Females influence the fitness of their progeny according to whether they space eggs out and minimise competition between larvae, or they distribute their eggs randomly or in a clump and suffer the loss of both quantity and quality of offspring that result (Giga and Smith, 1981, 1985). The critical question that arises is whether females can detect eggs already present.

Our results indicated that $S$. zeamais tended to cluster eggs on the grains. The $V / M$ ratios showed the increase in the incidence of egg aggregation that occurred as the number of grains available for oviposition increased $(V / M=7.08,9.76$ and 82.73 for 20,40 and 80 grains, respectively). The females aggregated more eggs on lower grain quantities than on higher grain quantities. Legg et al. (1987) have also noticed that the distribution of maize weevil eggs on maize kernels was aggregated. However, they showed that egg aggregation generally increased with increasing maize weevil density and duration of oviposition/feeding period, although the specific response depended on the grain density. This is in agreement with our results showing that the 
total number of eggs increased with the increasing number of grains available. Also, the number of utilised grains increased with the increase of the grain number, and consequently the number of emerged adults.

According to Smith (1986) and Fava and Springhetti (1991) Sitophilus females do not mark the grain where they have laid their eggs and therefore they are unable to recognise the presence of eggs, and the careful sealing in of an egg with a protein plug helps to hide the oviposition puncture. This kind of egg laying is surprising because it is not clear how S. zeamais benefits from laying more eggs in the same grains. If the tendency to clump eggs within grains does not depend on experimental conditions, some adaptive meaning could be postulated. At first sight such a trait would seem disadvantageous, since only one larvae per grain develops normally, and therefore a uniform or even a random egg distribution could lead to more offspring. However, cannibalism occurring within grains could enhance the survival of cannibalistic larvae; hence this compensates for reproductive costs and ultimately increases the fitness. This hypothesis is supported by the fact that the quality of survivors is not reduced by competition, for example emergence weight of the emerged adults and sex ratio. Neither factor differed significantly among treatments, although more eggs were laid and more adults emerged in the jars of higher grain quantity than in jars of lower grain quantity. Destruction of eggs laid by other weevils could be an additional strategy to cannibalism. Generally, weevil larvae are not able to migrate between grains and larval competition is very high in a host grain with multiple larvae. Either the Sitophilus female should attempt to reduce the individual competition faced by its offspring by killing eggs already present (ovicidal strategy), or a larva within a grain should destroy other larvae (attack strategy) to select for maximum fitness (Smith and Lessells, 1985). However, there are no published estimates of the levels of ovicide and larvicide in Sitophilus species. Further results and analyses are needed to test this hypothesis.

In West Africa, maize farmers generally store shelled maize kernels or unshelled cobs on a small scale. This method of small scale on-farm storage allows farmers to store sufficient maize for home consumption and the current results could predict that a lower level of weevil infestation may occur in such traditional maize storage than in larger communal facilities.

\section{Acknowledgements}

We are grateful to Professor Jean-Jacques Claustriaux and Mr Jose Villaroel for their advice on the choice of the statistical tests. We also thank anonymous referees for critically reviewing during the preparation of this manuscript. This research was supported by a Ph.D. grant from DGCI to Mathias Danho.

\section{References}

Dobie, P., 1974. The laboratory assessment of the inherent susceptibility of maize varieties to post-harvest infestation by Sitophilus zeamais Motsch. (Coleoptera, Curculionidae). Journal of Stored Products Research 10, $183-187$.

Fava, A., Springhetti, A., 1991. Egg distribution of Sitophilus granarius L. (Col., Curculionidae) in Triticum aestivum caryopses. Journal of Applied Entomology 111, 406-411. 
Frankenfeld, J.C., 1948. Staining methods for detecting weevil infestation in grain. USDA Bulletin of Entomology R Q. E-T. Series 256, 1-4.

Giga, D.P., Smith, R.H., 1981. Varietal resistance and intraspecific competition in the cowpea weevils Callosobruchus maculatus and C. chinensis (Coleoptera: Bruchidae). Journal of Applied Ecology 18, 755-761.

Giga, D.P., Smith, R.H., 1985. Oviposition markers in Callosobruchus maculatus (F.) and C. rhodesianus (Pic) (Coleoptera, Bruchidae): asymmetry of interspecific responses. Agriculture, Ecosystems and Environment 12, 229-233.

Halstead, D.G.H., 1963. External sex differences in stored products Coleoptera. Bulletin of Entomological Research 54, 119-134.

Holloway, G.J., 1984. Genetic differentiation and life history variation in the rice weevil, Sitophilus oryzae (L.). Ph.D. Thesis, University of Reading.

Honek, A., 1993. Intraspecific variation in body size and fecundity of insects: a general relationship. Oïkos 67, 483-492.

Legg, D.E., Barney, R.J., Tipping, P.W., Rodriguez, J.G., 1987. Factors influencing the distribution of maize weevil (Coleoptera: Curculionidae) eggs on maize. Environmental Entomology 16, 809-813.

Longstaff, B.C., 1981. Biology of the grain pest species of the genus Sitophilus (Coleoptera: Curculionidae): a critical review. Protection Ecology 2, 82-130.

Markham, R.H., Bosque-Pérez, N.A., Borgemeister, C., Meikle, W.G., 1994. Developing pest management strategies for the maize weevil, Sitophilus zeamais and the larger grain borer, Prostephanus truncatus, in the humid and subhumid tropics. FAO Plant Protection Bulletin 42.

Myers, J.H., 1978. Selecting a measure of dispersion. Environmental Entomology 7, 619-621.

Povey, S.R., Sibly, R.H., 1992. No oviposition plasticity in Sitophilus oryzae. Journal of Stored Products Research 28, $11-14$.

Richards, O.W., 1947. Observations on grain-weevils Calandra (Coleoptera: Curculionidae). I. General biology and oviposition. Proceedings of the Zoological Society of London 117, 1-43.

Schoonhoven, A.V., Horber, E., Mills, R.B., 1976. Conditions modifying expression of resistance of maize kernels to the maize weevil. Environmental Entomology 5, 163-168.

Segrove, F., 1951. Oviposition behaviour in the two strains of the rice weevil, Calandra oryzae Linn. (Coleoptera: Curculionidae). Journal of Experimental Biology 28, 281-297.

Sharifi, S., 1972. Oviposition site and egg plug staining as related to development of two species of Sitophilus in wheat kernels. Zeitschrift für Angewandte Entomologie 71, 428-431.

Smith, R.H., 1986. Oviposition, competition and population dynamics in storage insects. Proceedings of the 4th International Working Conference on Stored-Product Protection, Tel Aviv, Israel, September, pp. 427-433.

Smith, R.H., Lessells, C.M., 1985. Oviposition, ovicide and larval competition in granivorous insects. In: Sibly, R.M., Smith, R.H. (Eds.), Behavioural Ecology: Consequences of Adaptive Behaviour. Blackwell Scientific, Oxford, pp. 423-448.

Stejskal, V., Kucerova, Z., 1996. The effect of grain size on the biology of Sitophilus granarius L. (Coleoptera: Curculionidae). I. Oviposition, distribution of eggs and adult emergence. Journal of Applied Entomology 120, $143-146$.

Throne, J.E., 1994. Life history of immature maize weevils (Coleoptera: Curculionidae) on corn stored at constant temperatures and relative humidities in the laboratory. Environmental Entomology 23, 1459-1471.

Walgenbach, C.A., Burkholder, W.E., 1987. Mating behaviour of the maize weevil Sitophilus zeamais (Coleoptera: Curculionidae). Annals of the Entomological Society of America 80, 578-583.

Walgenbach, C.A., Phillips, J.K., Faustini, D.L., Burkholder, W.E., 1983. Male-produced aggregation pheromone of the maize weevil, Sitophilus zeamais, and interspecific attraction between three Sitophilus species. Journal of Chemical Ecology 9, 831-841.

Widstrom, N.M., McMillian, W.W., Wiseman, B.R., 1978. Improving effectiveness of measurements for seed resistance to maize weevil. Journal of Economic Entomology 71, 901-903. 